Laser Chem., Vol. 16, pp. 139-149

Reprints available directly from the publisher

Photocopying permitted by license only
(C) 1996 OPA (Overseas Publishers Association) Amsterdam B.V. Published in The Netherlands by Harwood Academic Publishers GmbH Printed in Malaysia

\title{
INHIBITION EFFECT ON MOLECULAR WEIGHT DISTRIBUTION GENERATED UNDER FREE RADICAL POLYMERIZATION BY LASER PULSES
}

\author{
A.V. EVSEEV and A.N. NIKITIN \\ Research Centre for Technological Lasers, Academy of Science of Russia, \\ Shatura, Moscow Region, 140700, Russia
}

(Received 21 March, 1995)

\begin{abstract}
Molecular weight distributions (MWDs) under free radical polymerization initiation by an arbitrary sequence of radiation pulses have been investigated. Analytical expressions enabling to account for influence of the polymer chains linear termination (for example, inhibition) have been obtained. On the basis of these expressions the MWDs generated by pulse-periodic radiation have been investigated theoretically. The calculated MWDs for polymerization in the presence of high concentration of retarder have been given and the influence of the inhibitor burning within the irradiation process on the MWDs character have been studied. It has been shown that possibility to employ the PLP method for determination of polymerization rate constant $\left(\mathrm{k}_{\mathrm{p}}\right)$ depends significantly on the efficiency of chains linear termination: the PLP method can be confidently used at $\tau>\mathrm{T}$ (where $\tau$ is the effective lifetime of growing radicals, $\mathrm{T}$ is the pulse irradiation period) and it is doubtful if $\tau<\mathrm{T} / 3$. The expression for specifying of pulses number required for establishment of pseudostationary polymerization has been obtained for the polymerization at high concentration of retarder. The calculated MWDs for polymerization of MMA are presented.
\end{abstract}

KEY WORDS: Photopolymerization, Pulsed laser polymerization, Radical polymerization, Molecular weight distribution, Inhibition, Retardation.

\section{INTRODUCTION}

At present the Pulsed Laser Polymerization (PLP) method is used intensively for direct measurement of the propagation rate coefficient $\left(\mathrm{k}_{\mathrm{p}}\right){ }^{1-3}$ The essence of this method is initiation of polymerization by periodic sequence of short laser pulses and investigation of MWD due to irradiation with gel-penetrating chromatography ${ }^{4-9}$ or with time-offlight mass-spectrometry. ${ }^{10}$ By pulse-periodic radiation the MWD feature peaks equally spaced in MWD spectrum, the distance between peaks is equal to $\mathrm{k}_{\mathrm{p}}[\mathrm{M}] \mathrm{T}$, where $[\mathrm{M}]$ is the monomer concentration and $\mathrm{T}$ is the irradiation period. Currently a series of experiments considering the MWDs obtained by pulse-periodic radiation has been fulfilled. ${ }^{4-10}$ The experimental results have shown that employing the PLP is not necessarily successful. Thus, determination of applicability limits for this method and obtaining simple relationships which would enable to model the MWDs spectra and to specify their base features are of some interest.

In our earlier paper ${ }^{11}$ for simple scheme of radical polymerization which took into account only reactions of polymer chains initiation, propagation and termination by 
recombination of radicals and their disproportionation, the analytical expressions were obtained for calculation of distributions of growing and "dead" macromolecules along the chains length under polymerization initiation by arbitrary sequence of radiation pulses. The MWDs generated by polymerization initiation with pulse-periodic and periodic sets of short pulses were caluculated for insignificant monomer-to-polymer conversion on the assumption that the Flory principle ${ }^{12}$ is satisfied and the constant of chain initiation rate $\left(\mathrm{k}_{\mathrm{i}}\right)$ is more greater or equal to the $\mathrm{k}_{\mathrm{p}}$ value.

The effect of repetition rate and initiating pulses fluence as well as rate constants of chain propagation and termination on the MWDs character was investigated. It was also shown that the solution of the MWD problem derived for polymerization initiated by $\delta$-shape pulses may be used for definition of MWDs both under polymerization initiated by pulses of any form and duration and under continuous radiation at non quasistationary process of polymerization.

The present paper studies theoretically the MWDs for polymerization scheme taking into account the inhibition reaction. The polymerization inhibitors, as a rule, are presented in the initial monomer even after special cleaning operation. Thus the effect of inhibitor on the polymerization process and hence on the MWDs always takes place in one way or another. The efficiency of inhibition is specified by relationship between rates of inhibition and polymerization $\left(\mathrm{k}_{\mathrm{t}}^{\prime}[\mathrm{Z}] / \mathrm{k}_{\mathrm{p}}[\mathrm{M}]\right.$, where $\mathrm{k}_{\mathrm{t}}^{\prime}$ is the rate constant of inhibition reaction and $[Z]$ is the inhibition concentration) and by rather low efficiency polymerization is performed enough successfully (in case of retardation). So far the influence of inhibition reaction on the MWDs was not studied for the case of free radical polymerization initiated by pulse-periodic irradiation. This reaction, as shown later, can change the MWDs character significantly and, for example, substantially limit the possibility for application of the PLP method for specifying the $k_{p}$ value.

\section{POLYMERIZATION MODEL AND SOLUTION OF KINETIC EQUATIONS}

As addition to reactions of initiation, propagation and termination by combination and disproportionation being considered in article ${ }^{11}$ the present paper takes into account the rection of growing chains termination due to interaction between radicals and inhibitor $[\mathrm{Z}]$ solved in the monomer:

$$
\mathrm{XM}_{\mathrm{i}}+\mathrm{Z} \stackrel{\mathrm{k}_{\mathrm{t}}^{\prime}}{\longrightarrow} \mathrm{XM}_{\mathrm{i}} \mathrm{Z}
$$

where $\mathrm{XM}_{\mathrm{i}}$ are the growing radicals of length $\mathrm{i} ; \mathrm{XM}_{\mathrm{i}} \mathrm{Z}$ is considered as a dead polymer of length $i$.

In term (1) and with the same assumptions as in paper ${ }^{11}$ the system of kinetic equations for concentrations of growing $\left(A_{i}\right)$ and dead macromolecules $\left(B_{i}\right)$ takes the form:

$$
\begin{aligned}
& \frac{d A_{0}}{d t}=-k_{p} A_{0}[M]-k_{t} A_{0} \sum_{j=0}^{\infty} A_{j}-k_{t}^{\prime} A_{0}[Z], \\
& \frac{d A_{i}}{d t}=k_{p} A_{i-1}[M]-k_{p} A_{i}[M]-k_{t} A_{i} \sum_{j=0}^{\infty} A_{j}-k_{t}^{\prime} A_{i}[Z],
\end{aligned}
$$




$$
\frac{d B_{i}}{d t}=0.5 k_{t c} \sum_{j=0}^{i} A_{j} A_{i-j}+k_{t d} A_{i} \sum_{j=0}^{\infty} A_{j}+k_{t}^{\prime} A_{i}[Z],
$$

where $\mathrm{k}_{\mathrm{tc}}$ and $\mathrm{k}_{\mathrm{td}}$ are the rate constants of radicals combination and disproportionation reactions respectively, $\mathrm{k}_{\mathrm{t}}=\mathrm{k}_{\mathrm{tc}}+\mathrm{k}_{\mathrm{td}}$.

Under initiation of polymerization by arbitrary sequence of $\delta$-shape pulses $1,2, \ldots \mathrm{N}$ which generate radical concentrations $\mathrm{W}_{1}, \mathrm{~W}_{2}, \ldots \mathrm{W}_{\mathrm{N}-1}, \mathrm{~W}_{\mathrm{N}}$ in medium and follow one after another at intervals $\mathrm{T}_{1}, \mathrm{~T}_{2}, \ldots \mathrm{T}_{\mathrm{N}-2}, \mathrm{~T}_{\mathrm{N}-1}$ respectively, in supposition that the inhibitor concentration between $\mathrm{kth}$ and $(\mathrm{k}+1)$ th $(\mathrm{k}=1,2, \ldots, \mathrm{N}-1)$ pulses is not changed and equals to $\left[Z_{k}\right]$, the system of equations (2)-(4) posseses an analytical solution:

$$
\begin{aligned}
\mathrm{A}_{\mathrm{i}}^{(\mathrm{N})}= & \varphi_{\mathrm{N}}(\mathrm{t}) \sum_{\mathrm{j}=1}^{\mathrm{N}} \beta_{\mathrm{j}}^{\mathrm{N}} \rho_{\mathrm{i}}\left(\mathrm{Q}_{\mathrm{j}}^{\mathrm{N}}+\mathrm{t}\right) \\
\mathrm{B}_{\mathrm{i}}^{(\mathrm{N})}= & 0.5 \mathrm{k}_{\mathrm{tc}} \sum_{\mathrm{r}=1}^{\mathrm{N}-1} \sum_{1, \mathrm{~m}=1}^{\mathrm{r}} \beta_{1}^{\mathrm{r}} \beta_{\mathrm{m}}^{\mathrm{r}} \int_{0}^{\mathrm{T}_{\mathrm{r}}} \varphi_{\mathrm{r}}^{2}(\mathrm{v}) \rho_{\mathrm{i}}\left(\mathrm{Q}_{1}^{\mathrm{r}}+\mathrm{Q}_{\mathrm{m}}^{\mathrm{r}}+2 \mathrm{v}\right) \mathrm{dv} \\
& +0.5 \mathrm{k}_{\mathrm{tc}} \sum_{1, \mathrm{~m}=1}^{\mathrm{N}} \beta_{1}^{\mathrm{N}} \beta_{\mathrm{m}}^{\mathrm{N}} \int_{0}^{\mathrm{t}} \varphi_{\mathrm{N}}^{2}(\mathrm{v}) \rho_{\mathrm{i}}\left(\mathrm{Q}_{1}^{\mathrm{N}}+\mathrm{Q}_{\mathrm{m}}^{\mathrm{N}}+2 \mathrm{v}\right) \mathrm{dv} \\
& +\mathrm{k}_{\mathrm{td}} \sum_{\mathrm{r}=1}^{\mathrm{N}-1} \mathrm{~F}_{1}^{\mathrm{r}}(0) \sum_{1=1}^{\mathrm{r}} \beta_{1}^{\mathrm{r}} \int_{0}^{\mathrm{T}_{\mathrm{r}}} \varphi_{\mathrm{r}}^{2}(\mathrm{v}) \rho_{\mathrm{i}}\left(\mathrm{Q}_{1}^{\mathrm{r}}+\mathrm{v}\right) \mathrm{dv} \\
& +\mathrm{k}_{\mathrm{td}} \mathrm{F}_{1}^{\mathrm{N}}(0) \sum_{1=1}^{\mathrm{N}} \beta_{1}^{\mathrm{N}} \int_{0}^{\mathrm{t}} \varphi_{\mathrm{N}}^{2}(\mathrm{v}) \rho_{\mathrm{i}}\left(\mathrm{Q}_{1}^{\mathrm{N}}+\mathrm{v}\right) \mathrm{dv} \\
& +\sum_{\mathrm{r}=1}^{\mathrm{N}-1} \frac{1}{\tau_{\mathrm{r}}} \sum_{1=1}^{\mathrm{r}} \beta_{1}^{\mathrm{r}} \int_{0}^{\mathrm{T}_{\mathrm{r}}} \varphi_{\mathrm{r}}(\mathrm{v}) \rho_{\mathrm{i}}\left(\mathrm{Q}_{1}^{\mathrm{r}}+\mathrm{v}\right) \mathrm{dv}+\frac{1}{\tau_{\mathrm{N}}} \sum_{1=1}^{\mathrm{N}} \beta_{1}^{\mathrm{N}} \int_{0}^{\mathrm{t}} \varphi_{\mathrm{N}}(\mathrm{v}) \rho_{\mathrm{i}}\left(\mathrm{Q}_{1}^{\mathrm{N}}+\mathrm{v}\right) \mathrm{dv},
\end{aligned}
$$

where

$$
\begin{aligned}
\mathrm{p}_{\mathbf{k}}(\lambda) & =\frac{(\mathrm{U} \lambda)^{\mathrm{k}} \exp (-\mathrm{U} \lambda)}{\mathrm{k} !}, \\
\varphi_{\mathrm{k}}(\lambda) & =\frac{\exp \left(-\lambda / \tau_{\mathrm{k}}\right)}{1+\mathrm{k}_{\mathrm{t}} \tau_{\mathrm{k}} \mathrm{F}_{1}^{\mathrm{k}}(0)\left(1-\exp \left(\lambda / \tau_{\mathrm{k}}\right)\right)}, \\
\mathrm{F}_{1}^{\mathrm{k}}(0) & =\mathrm{W}_{\mathrm{k}}+\mathrm{F}_{1}^{\mathrm{k}-1}(0) \varphi_{\mathrm{k}-1}\left(\mathrm{~T}_{\mathrm{k}-1}\right), \\
\beta_{\mathrm{m}}^{\mathrm{k}} & =\mathrm{W}_{\mathrm{k}-\mathrm{m}+1} \prod_{\mathrm{r}=1}^{\mathrm{m}-1} \varphi_{\mathrm{k}-\mathrm{r}}\left(\mathrm{T}_{\mathrm{k}-\mathrm{r}}\right), \\
\mathrm{Q}_{\mathrm{m}}^{\mathrm{k}} & =\sum_{\mathrm{r}=1}^{\mathrm{m}-1} \mathrm{~T}_{\mathrm{k}-\mathrm{r}}, \\
\tau_{\mathrm{k}} & =1 / \mathrm{k}_{\mathrm{t}}^{\prime}\left[\mathrm{Z}_{\mathrm{k}}\right] \text { and } \mathrm{U}=\mathrm{k}_{\mathrm{p}}[\mathrm{M}] .
\end{aligned}
$$

The technique for solution of system of equations (2)-(4) is analogous to that described in paper ${ }^{11}$ and is not shown here. The radicals distribution at any time $t$ after the $N$ th pulse $\left(A_{i}^{(N)}\right)$ comprises a sum of $N$ group of radicals having Poison distribution $\left(\rho_{j}\left(Q_{j}^{(N)}+t\right)\right)$. Coefficients $\beta_{\mathrm{j}}^{\mathrm{k}}$ present the concentration of radicals produced by the $(\mathrm{k}-\mathrm{j}+1)$ th pulse 
and remaining by the time of the kth pulse. $Q_{j}^{k}$ is the time interval between $(k-j+1)$ th and kth pulses. $F_{1}^{(k)}(0)$ is the total radical concentration after the kth pulse. $\varphi_{k}(\lambda)$ features the reduction in radicals total concentration after the $\mathrm{kth}$ pulse both of inhibition $\left(\exp \left(-\lambda / \tau_{\mathrm{k}}\right)\right)$ and of chains termination $\left(\left(1+\mathrm{k}_{\mathrm{t}} \tau_{\mathrm{k}} \mathrm{F}_{1}^{(\mathbf{k})}(0)\left(\left(1-\exp \left(-\lambda / \tau_{\mathrm{k}}\right)\right)\right)^{-1}\right)\right.$.

In expression for concentration of dead polymer of length $i$, the odd numbered terms represent concentrations of polymer molecules generated by the time when the Nth pulse arises by combination, disproportionation and inhibition respectively, and the even numbered terms describe the change in concentration of polymer molecules in time caused by reactions after the Nth pulse.

Integrating the equation

$$
\frac{\mathrm{d}[\mathrm{M}]}{\mathrm{dt}}=-\mathrm{k}_{\mathrm{p}}[\mathrm{M}] \sum_{\mathbf{j}=0}^{\infty} \mathrm{A}_{\mathbf{j}}
$$

at low monomer-to-polymer conversion after the Nth pulse for monomer concentration we obtain:

$$
\begin{aligned}
{\left[\mathrm{M}^{(\mathrm{N})}\right]=} & {\left[\mathrm{M}^{(\mathrm{N}-1)}\right]-\frac{\mathrm{U}}{\mathrm{k}_{\mathrm{t}}} \ln \left(1+\mathrm{k}_{\mathrm{t}} \tau_{\mathrm{N}} \mathrm{F}_{1}{ }^{(\mathrm{N})}(0)\left(1-\exp \left(-\mathrm{t} / \tau_{\mathrm{N}}\right)\right)\right) } \\
= & {\left[\mathrm{M}^{(0)}\right]-\sum_{\mathrm{j}=1}^{\mathrm{N}-1} \frac{\mathrm{U}}{\mathrm{k}_{\mathrm{t}}} \ln \left(1+\mathrm{k}_{\mathrm{t}} \tau_{\mathrm{j}} \mathrm{F}_{1}{ }^{(\mathrm{j})}(0)\left(1-\exp \left(-\mathrm{T}_{\mathrm{j}} / \tau_{\mathrm{j}}\right)\right)\right) } \\
& -\frac{\mathrm{U}}{\mathrm{k}_{\mathrm{t}}} \ln \left(1+\mathrm{k}_{\mathrm{t}} \tau_{\mathrm{N}} \mathrm{F}_{1}^{(\mathrm{N})}(0)\left(1-\exp \left(-\mathrm{t} / \tau_{\mathrm{N}}\right)\right)\right)
\end{aligned}
$$

This expression was used for calculation monomer-to-polymer conversion.

\section{CALCULATION RESULTS AND DISCUSSION}

Expression (5)-(6) for $\mathrm{A}_{\mathrm{i}}^{(\mathrm{N})}$ and $\mathrm{B}_{\mathrm{i}}^{(\mathrm{N})}$ were used for investigation of distributions generated by pulse -periodic irradiation $\left(T_{j}=T, j=1,2, \ldots\right)$. It was supposed that each pulse produces initiating radicals within polymerizing medium instantaneously and the initiator concentration is not changed by polymerization process. Thus the initiating radicals concentration for $j=1,2, \ldots$ is considered as $W_{j}=W$.

\section{Polymerization at high inhibitor concentration $\left([\mathrm{Z}] \gg \mathrm{W}\right.$ and $\left.\tau_{\mathrm{k}}=\tau\right)$}

By pulse-periodic radiation the time dependence of radicals total concentration $R(t)$ is written as:

$$
\mathrm{R}(\mathrm{t})=\mathrm{F}_{1}{ }^{(\mathrm{N})}(0) \varphi_{\mathrm{N}}(\mathrm{t})
$$

where $(\mathrm{N}-1) \mathrm{T} \leqslant \mathrm{t} \leqslant \mathrm{NT}, \mathrm{N}=1,2, \ldots$, and $\mathrm{T}=\mathrm{f}^{-1} . \mathrm{F}_{1}(0)$ is determined by recurrence (9). Figure 1 depicts time dependence of $R(t) / W$ calculated for different $\tau$. The lower dependence is typical for inhibition polymerization where $T \gg \tau$ and the medium is practically devoid of growing radicals by the time when the next radical group arises. In this case the pseudostationary polymerization regime ${ }^{1}$ is established following the 


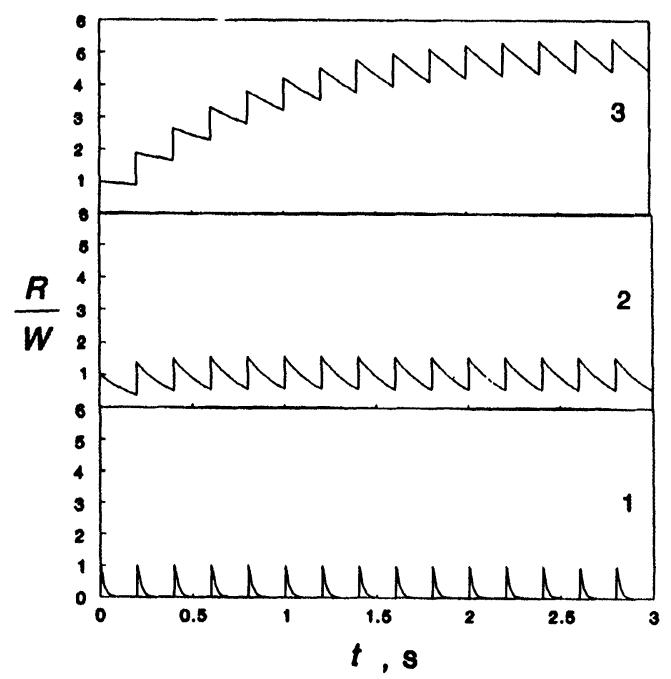

Figure 1 Time dependences of radicals relative concentrations ( $R / \mathrm{W}$ versus $\mathrm{t})$ calculated for $\tau=0.02$ $(1), 0.2(2), 2(3) \mathrm{s}, \mathrm{k}_{\mathrm{t}}=10^{7} 1 /(\mathrm{mol} \cdot \mathrm{s}), \mathrm{W}=10^{-7} \mathrm{~mol} / \mathrm{l}$ and $\mathrm{T}=0.2 \mathrm{~s}$.

second pulse; the change in monomer concentration is given by simple relation

$$
[\mathrm{M}] /\left[\mathrm{M}^{(0)}\right]=1-\mathrm{N} \omega,
$$

where $\omega$ is the polymer yield per pulse

$$
\omega=\frac{\mathrm{k}_{\mathrm{p}}}{\mathrm{k}_{\mathrm{t}}} \ln \left(1-\mathrm{k}_{\mathrm{t}} \tau \mathrm{W}\right)
$$

In cases 2 and 3 the radical pseudostationary concentration is established following some pulses number dependent on $\tau$. Taking into account the inhibition the maximal radicals concentration $R_{m}^{p s}$ at pseudostationary state specificed as in paper ${ }^{1}$ is given as:

$$
\mathrm{R}_{\mathrm{m}}^{\mathrm{ps}}=-\frac{1-\mathrm{W} \tau \mathrm{k}_{\mathrm{t}}}{2 \tau \mathrm{k}_{\mathrm{t}}}+\frac{1+\mathrm{W} \tau \mathrm{k}_{\mathrm{t}}}{2 \tau \mathrm{k}_{\mathrm{t}}}\left(1+\frac{4 \mathrm{~W} \tau \mathrm{k}_{\mathrm{t}} \exp (-\mathrm{T} / \tau)}{(1-\exp (-\mathrm{T} / \tau))\left(1+\mathrm{W} \tau \mathrm{k}_{\mathrm{t}}\right)^{2}}\right)^{1 / 2}
$$

The pulses number $\left(\mathrm{N}_{\varepsilon}^{\mathrm{ps}}\right)$ required for establishment of pseudostationary state can be determined in the following way. The pseudostationary state is established at time $t_{p s}$ after the first laser pulse when the contribution of radicals, remained after the first pulse, to the total concentration can be neglected at the precision $\varepsilon$. Since radical lifetime is about $\left(\mathrm{k}_{\mathrm{t}} \mathrm{R}_{\mathrm{av}}+\tau^{-1}\right)^{-1}$ it can be written

$$
\mathrm{t}_{\mathrm{ps}}=\mathrm{N}_{\varepsilon}^{\mathrm{ps}}=\mathrm{a}_{\varepsilon}\left(\mathrm{k}_{\mathrm{t}} \mathrm{R}_{\mathrm{av}}+\mathrm{b}_{\varepsilon} \tau^{-1}\right)^{-1},
$$

where $\mathrm{a}_{\varepsilon}$ and $\mathrm{b}_{\varepsilon}$ are the parameters dependent on $\varepsilon$. The average radicals concentration $\mathbf{R}_{\mathrm{av}}$ of pseudostationary state is determined from the condition when the initiation 
velocity $\left(v_{i n}\right)$ equals that of chain termination $\left(v_{t}\right)$. Since

$$
\begin{aligned}
\mathrm{v}_{\mathrm{in}} & \approx \mathrm{W} / \mathrm{T}, \\
\mathrm{v}_{\mathrm{t}} & \approx \mathrm{k}_{\mathrm{t}} \mathrm{R}_{\mathrm{av}}^{2}+\mathrm{R}_{\mathrm{av}} / \tau,
\end{aligned}
$$

it can be written as

$$
\mathrm{R}_{\mathrm{av}} \approx-\left(2 \tau \mathrm{k}_{\mathrm{t}}\right)^{-1}+\left(2 \tau \mathrm{k}_{\mathrm{t}}\right)^{-1}\left(1+\mathrm{Wk}_{\mathrm{t}} \tau^{2} \mathrm{~T}^{-1}\right)^{1 / 2}
$$

From (18) and (21) we have

$$
\mathrm{N}_{\varepsilon}^{\mathrm{ps}} \approx \frac{2 \tau \mathrm{a}_{\varepsilon}}{\mathrm{T}\left(2 \mathrm{~b}_{\varepsilon}-1\right)+\left(\mathrm{T}^{2}+4 \mathrm{k}_{\mathrm{t}} \mathrm{WT} \tau^{2}\right)^{1 / 2}}
$$

Without inhibition ( $\tau$ tends to infinity) from (22) we have $\mathrm{N}_{\varepsilon}^{\mathrm{ps}} \approx \mathrm{a}_{\varepsilon}\left(\mathrm{k}_{\mathrm{t}} \mathrm{WT}\right)^{-1 / 2}$ which corresponds to the result in paper $^{11}$. It was obtained that at $\varepsilon=0.1 \mathrm{a}_{\varepsilon} \approx 1.47$ and $\mathrm{b}_{\varepsilon} \approx 0.64$; at $\varepsilon=0.01 \mathrm{a}_{\varepsilon} \approx 2.65$ and $\mathrm{b}_{\varepsilon} \approx 0.58$; at $\varepsilon=0.001 \mathrm{a}_{\varepsilon} \approx 3.8$ and $\mathrm{b}_{\varepsilon} \approx 0.55$.

Consider changes in the MWDs character under pulse-periodic irradiation depending on $\tau=\left(k_{t}^{\prime}[Z]\right)^{-1}$ and laser pulse repetition rate $\left(f=T^{-1}\right)$. Simultaneously discuss the information on polymerization kinetic parameters which can be obtained from the MWDs spectra at pulse-periodic irradiation.

\section{a) Retarder}

Figure 2 shows mass MWDs $\left(\mathrm{iB}_{\mathrm{i}}\right)$ calculated for $\mathrm{t}=30 \mathrm{~s}$ after the 50 th pulse in the case of chain termination by radical combination (a) or their disproportionation (b). For all distributions $\mathrm{N}_{\varepsilon}^{\mathrm{ps}} \leqslant 5$ thus the character of each distribution does not change essentially just after the 5 th pulse. The parameters (pulse repetition rate, and radicals concentration generated by each pulse) are chosen so that the MWDs spectrum has 4 contrasting peaks without retarder. By decreasing $\tau$ which features the radicals lifetime associated with retardation, the intensity of high-molecular peaks reduces and the amplitude of lowmolecular ones increases. If the peaks are numbered from the origin of the coordinates the $n$th peak corresponding to the chain length $\mathrm{i}=\mathrm{nUT}=\mathrm{nk}_{\mathrm{p}}[\mathrm{M}] \mathrm{T}$ is conserved at $\mathrm{t}>\mathrm{nT}$. The calculation showed that for $\tau$ values confirming to $\mathrm{nT} / 3<\tau<\ln \mathrm{T}$ the highly attenuated peak can be yet found, then at $\tau<\mathrm{nT} / 3$ the nth peak will be absent in $B_{i}$ distribution. It has been shown in paper $^{11}$ that at $\mathrm{UT}<10$ no pulse-periodic feature of polymerization initiation shows up in the MWDs spectrum for dead polymer. Thus at any repetition rate of initiating pulses the nth peak will be absent when $\tau U<n 10 / 3$. Hence, the high concentration of the retarder can cause the lack of extrema in the MWDs and thus it may account for inapplicability of the PLP method for determination of $\mathrm{k}_{\mathrm{p}}$ value.

Table 1 shows $i$ values for chain termination due to radicals combination (a) and their disproportionation (b). These values correspond to minima (min), maxima ( $\max$ ) and inflection points in $\mathrm{B}_{\mathrm{i}}$ distributions for 4 peaks present in the MWDs in Figure 2. Without retardation $\left(k_{t}^{\prime}=0\right)$ the difference between the neighboring maxima or minima both in $B_{i}$ distribution and in $\mathrm{dB}_{\mathrm{i}} / \mathrm{dt}$ is given as $\mathrm{UT}=\mathrm{k}_{\mathrm{p}}[\mathrm{M}] \mathrm{T}$ with accuracy better than $1 \%$ However, at contrast deterioration the method for UT determination by the inflection points put forward by paper ${ }^{1}$ is preferential because in the absence of extrema on values $i$ 


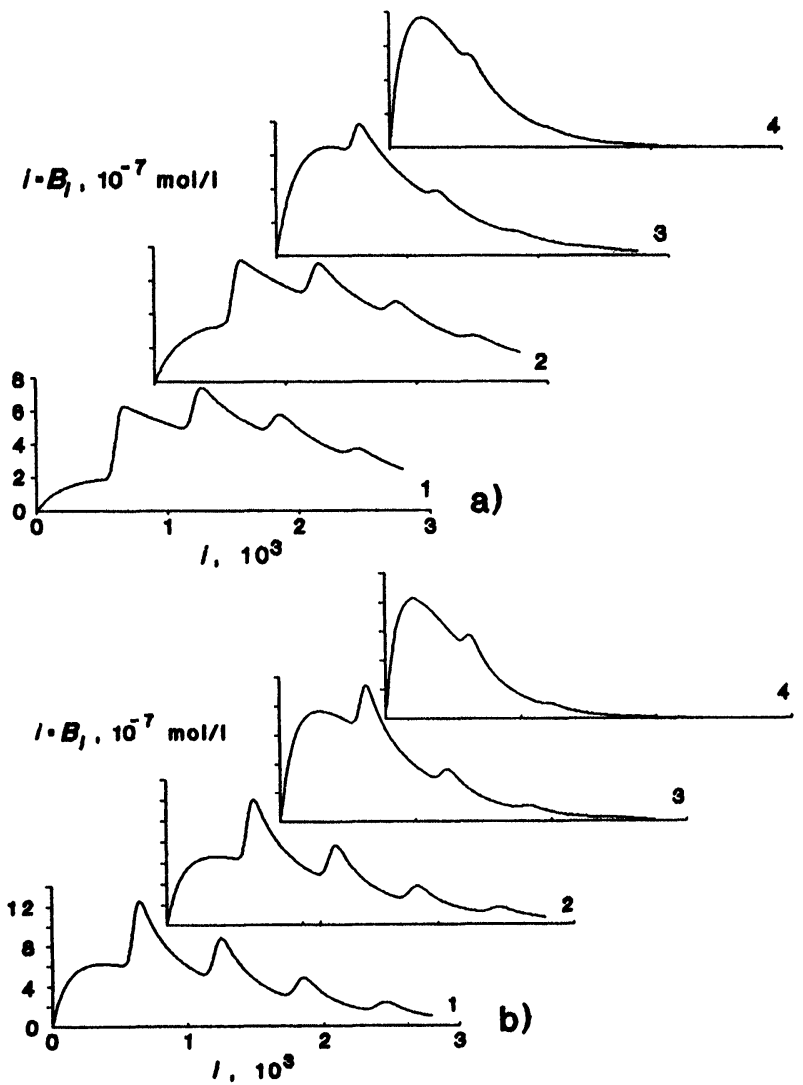

Figure 2 Distributions of $i B_{i}$ at $k_{t}^{\prime}=0(1), 1(2), 5(3)$ and $10(4) 1 /(\mathrm{mol} \cdot \mathrm{s})$ for chain termination by radicals combination (a) and their disproportionation (b) in $30 \mathrm{~s}$ after the 50th radiation pulse. Calculated for $\mathrm{k}_{\mathrm{p}}[\mathrm{M}]=300 \mathrm{~s}^{-1}, \mathrm{k}_{\mathrm{tc}}(\mathrm{a})=\mathrm{k}_{\mathrm{td}}(\mathrm{b})=10^{7} 1 /(\mathrm{mol} \cdot \mathrm{s}), \mathrm{W}=5 \cdot 10^{-8} \mathrm{~mol} / \mathrm{l} .[\mathrm{Z}]=0.1 \mathrm{~mol} / 1$ and $\mathrm{T}=2 \mathrm{~s}$.

Table 1 Minima (min), maxima (max) and inflection (inf) points in $B_{i}$ distributions corresponding to the distributions given in Figure 2a(a), in Figure $2 b(b)$ and in Figure 4(c).

\begin{tabular}{|c|c|c|c|c|c|c|c|c|c|c|c|c|c|}
\hline \multirow{2}{*}{\multicolumn{2}{|c|}{$n$}} & \multirow[b]{2}{*}{$\min$} & \multicolumn{2}{|c|}{1} & \multicolumn{3}{|c|}{2} & \multicolumn{3}{|c|}{3} & \multicolumn{3}{|c|}{4} \\
\hline & & & $\inf$ & $\max$ & $\min$ & inf & $\max$ & $\min$ & inf & $\max$ & $\min$ & inf & $\max$ \\
\hline \multirow{4}{*}{$\mathrm{a}$} & 1 & 539 & 598 & 646 & 1136 & 1196 & 1248 & 1740 & 1794 & 1848 & 2340 & 2393 & 2441 \\
\hline & 2 & 545 & 598 & 641 & 1143 & 1196 & 1241 & 1752 & 1793 & 1831 & 2386 & 2391 & 2396 \\
\hline & 3 & 566 & 596 & 624 & 1183 & 1194 & 1204 & - & 1793 & - & - & 2393 & - \\
\hline & 4 & - & 596 & - & - & 1194 & - & - & 1802 & - & - & 2417 & - \\
\hline & 1 & 547 & 596 & 633 & 1133 & 1193 & 1241 & 1723 & 1791 & 1846 & 2307 & 2389 & 2454 \\
\hline \multirow{3}{*}{$\mathrm{b}$} & 2 & 549 & 596 & 632 & 1136 & 1193 & 1238 & 1729 & 1791 & 1841 & 2324 & 2389 & 2443 \\
\hline & 3 & 559 & 595 & 625 & 1153 & 1192 & 1226 & 1753 & 1790 & 1822 & 2357 & 2388 & 2416 \\
\hline & 4 & 576 & 595 & 611 & - & 1192 & - & - & 1791 & - & - & 2391 & - \\
\hline & 1 & 539 & 598 & 646 & 1136 & 1196 & 1248 & 1740 & 1794 & 1848 & 2340 & 2393 & 2441 \\
\hline \multirow{3}{*}{\multicolumn{2}{|c|}{$\begin{array}{l}2 \\
3 \\
4\end{array}$}} & 539 & 598 & 646 & 1136 & 1196 & 1248 & 1741 & 1794 & 1842 & 2353 & 2392 & 2428 \\
\hline & & 543 & 598 & 644 & 1137 & 1196 & 1248 & 1741 & 1794 & 1842 & 2353 & 2392 & 2428 \\
\hline & & 543 & 598 & 644 & 1137 & 1196 & 1248 & 1741 & 1794 & 1842 & 2353 & 2392 & 2429 \\
\hline
\end{tabular}


corresponding to the inflection points, it is able to determine the $\mathrm{k}_{\mathrm{p}}$ value with satisfactory accuracy.

\section{b) Inhibitor}

At $\tau \ll \mathrm{T}$ (the calculation showed that at $\tau<\mathrm{T} / 4$ ) the radicals due to irradiation disappear virtually before the next pulse arises and do not practically affect on the MWDs character. In this case the distribution of growing radicals after the $\mathrm{Nth}$ pulse has the form

$$
\mathrm{A}_{\mathrm{i}}^{(\mathrm{N})} \approx \mathrm{W} \varphi_{1}(\mathrm{t}) \rho_{\mathrm{i}}(\mathrm{t})
$$

and at $\mathrm{t}=\mathrm{T}$ for dead polymer distribution we have

$$
\mathrm{B}_{\mathrm{i}}^{(\mathrm{N})} \approx \mathrm{WN} \tau^{-1} \int_{0}^{\mathrm{T}} \varphi_{1}(\mathrm{v}) \rho_{\mathrm{i}}(\mathrm{v}) \mathrm{dv} .
$$

Integrating by parts and considering the upper limit equal to infinity instead of $\mathrm{T}$ we obtain:

$$
\mathrm{B}_{\mathrm{i}}^{(\mathrm{N})} \approx \frac{\mathrm{NW}}{(1+\mathrm{U} \tau)}\left(\frac{\mathrm{U} \tau}{1+\mathrm{U} \tau}\right)^{i} .
$$

The same relationship is obtained when the system of equations (2)-(4) is solved in quasistationary approximation at the initiation rate $\mathrm{W} / \mathrm{T}$. Thus at $\tau \ll \mathrm{T}$ (practically at $\tau \leqslant \mathrm{T} / 4$ ) the MWDs of dead macromolecules are described by the common relationship for polymerization initiation both with pulsed and continuous radiation.

It follows from (24) that the slope of $\ln \left(\mathrm{B}_{\mathrm{i}}\right)$ dependence versus $\mathrm{i}$ is unaffected by the irradiation parameters and is determined by relationship $U \tau /(1+U \tau)$ which can be used for definition of $U \tau$ parameter.

\section{Consideration of changing in inhibitor concentration}

At $1 / \tau_{\mathrm{k}} \gg \mathrm{k}_{\mathrm{t}} \mathrm{W}$ the initiation radicals serve for inhibitor burning as a rule and its concentration reduces by about $\mathrm{W}$ in one irradiation period. The influence of inhibitor concentration change on the character of growing and dead macromolecules distributions was investigated for the case of insignificant change of relative inhibitor concentration $([\Delta \mathrm{Z}] /[\mathrm{Z}] \ll 1)$. Chnages in $[\mathrm{Z}]$ were considered in the following way. A set of equations

$$
\begin{aligned}
\mathrm{dF}_{1} / \mathrm{dt} & =-\mathrm{k}_{\mathrm{t}} \mathrm{F}_{1}^{2}-\mathrm{k}_{1}^{\prime} \mathrm{F}_{1}[\mathrm{Z}] \\
\mathrm{d}[\mathrm{Z}] / \mathrm{dt} & =-\mathrm{k}_{\mathrm{t}}^{\prime} \mathrm{F}_{1}[\mathrm{Z}]
\end{aligned}
$$

was solved for initial conditions after each Nth pulse $F_{1}^{(\mathbf{N})}(0)=W+F_{1}^{(N-1)}(T),\left[Z^{(N)}(0)\right]=$ $\left[Z^{(N-1)}(T)\right]$ and the change in inhibitor concentration in one irradiation period was determined. It was assumed that for each $\mathbf{N} \tau_{\mathrm{N}}=2\left\{\mathbf{k}_{\mathrm{t}}^{\prime}\left(\left[\mathrm{Z}^{(\mathrm{N})}(0)\right]+\left[\mathrm{Z}^{(\mathrm{N})}(\mathrm{T})\right]\right)\right\}^{-1}$ and this expression was used for calculation of $A_{i}^{(N)}$ and $B_{i}^{(N)}$ distributions from formulae (5)-(6).

Figure 3 shows $\mathrm{iB}_{\mathrm{i}}^{(\mathbf{N})}$ distributions estimated for $\mathrm{t}=30 \mathrm{~s}$ after the 100 th (1), 300th (2), 500th (3), 700th (4) and 1000th (5) pulses. For selected parameters the inhibitor burning takes place up to $\mathrm{N}=500$ approximately. By $\mathrm{N}$ increasing the inhibitor concentration 


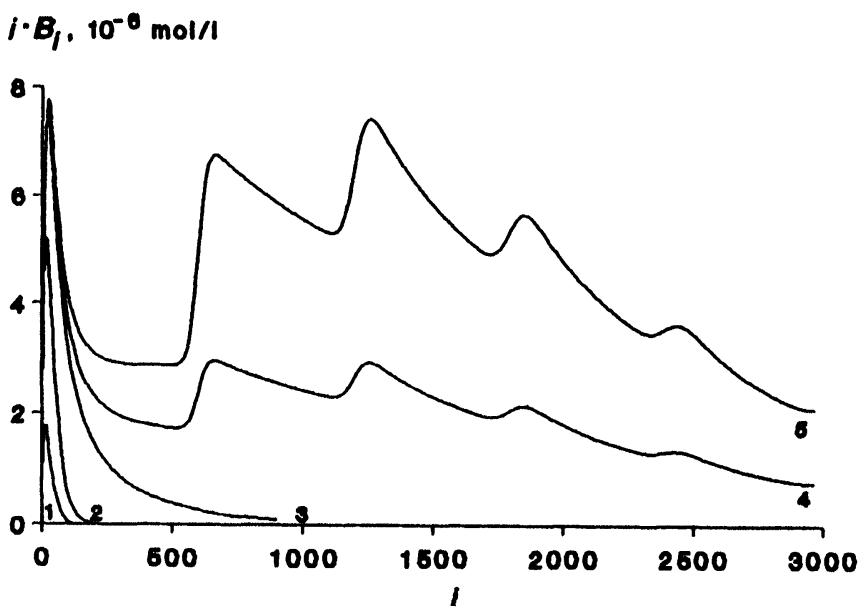

Figure 3 Distributions of $\mathrm{iB}_{\mathrm{i}}$ in $30 \mathrm{~s}$ after the 100th (1),300th (2),500th (3), 700th (4) and 1000th (5) radiation pulses for $\mathrm{k}_{\mathrm{p}}[\mathrm{M}]=300 \mathrm{~s}^{-1}, \mathrm{k}_{\mathrm{tc}}=10^{7} 1 /(\mathrm{mol} \cdot \mathrm{s}), \mathrm{k}_{\mathrm{td}}=0, \mathrm{~W}=5 \cdot 10^{-8} \mathrm{~mol} / \mathrm{l},[\mathrm{Z}]=2.5 \cdot 10^{-5} \mathrm{~mol} / 1$, $\mathrm{k}_{\mathrm{t}}^{\prime}=10^{6} 1 /(\mathrm{mol} \cdot \mathrm{s})$ and $\mathrm{T}=2 \mathrm{~s}$.

decreases, the average length of macromolecules increases $\left(\mathrm{i}_{\mathrm{av}} \approx \tau_{\mathrm{N}} \mathrm{U}\right)$ and the peaks are available in the MWDs spectrum (at $\tau_{\mathrm{N}}>\mathrm{T}$ ).

For investigation of burned inhibitor influence on the MWDs character and on the accuracy by polymerization rate constant determination, the MWDs spectra, formed at the conversion level equal to $1 \%$ for different initial inhibitor concentration, were calculated. Results of this calculation are give in Figure 4. The number of pulses required for obtaining the specified conversion increases with increasing $\left[Z_{0}\right]$. Only the low-molecular part of macromolecules distribution varies significantly as the inhibitor

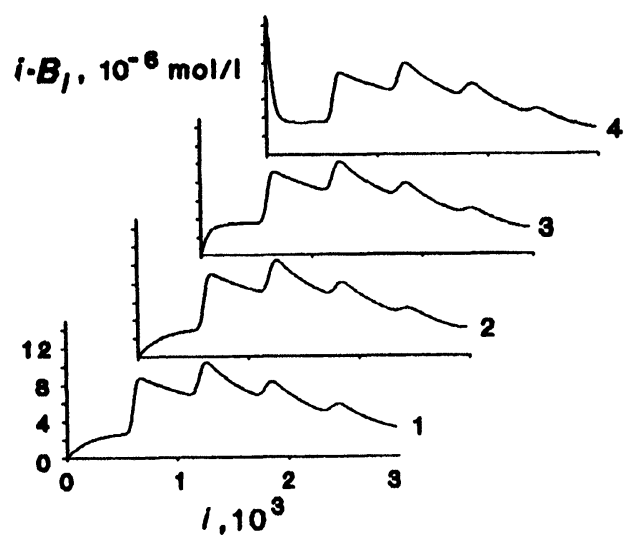

Figure 4 Distribution $\mathrm{iB}_{\mathrm{i}}$ for initial inhibitor concentration $\left[\mathrm{Z}_{0}\right]=0(1, \mathrm{~N}=694), 5 \cdot 10^{-7}(2, \mathrm{~N}=700)$, $5 \cdot 10^{-6}(3, \mathrm{~N}=780), 5 \cdot 10^{-5}(4, \mathrm{~N}=1667) \mathrm{mol} / 1$ after the monomer-to-polymer conversion equal to $1 \%$. Other parameters are similar to those in Figure 3. 

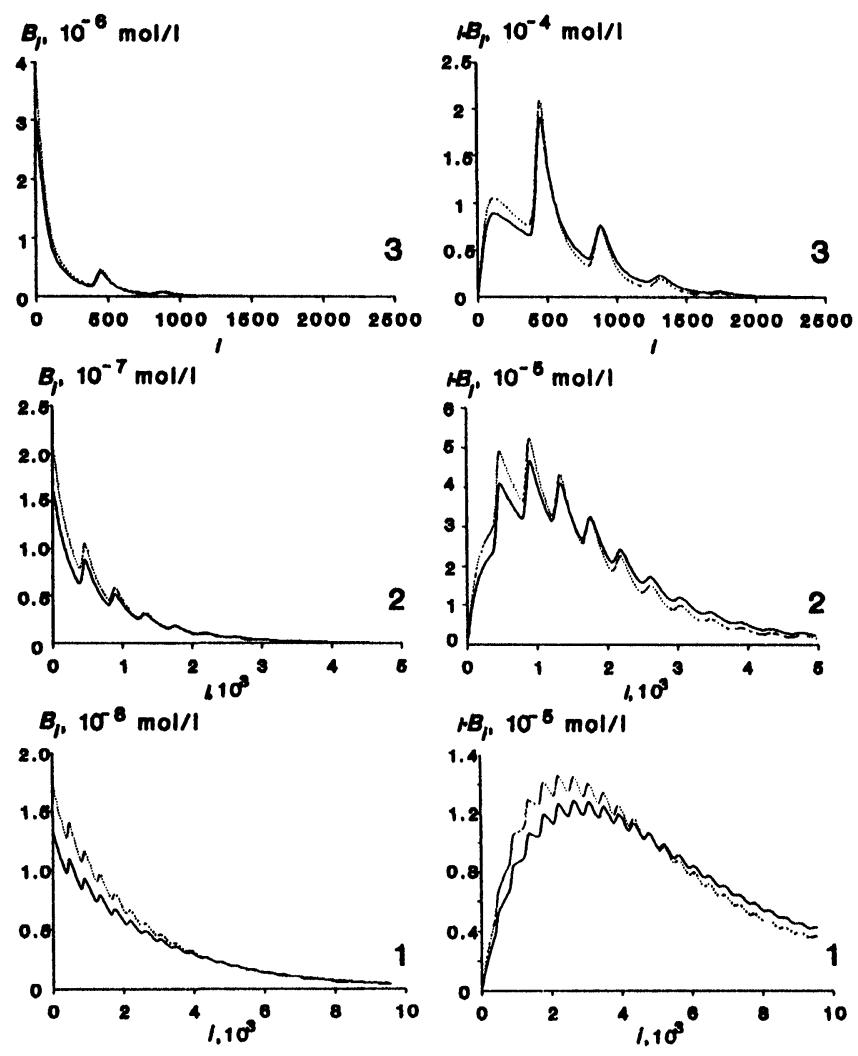

Figure 5 Distributions $B_{i}$ and $i B_{i}$ for bulk polymerization of methylmethacrylate at $f=10 \mathrm{~Hz}$ after the monomer-to-polymer conversion equal to $1 \%$. Calculated for $\mathrm{k}_{\mathrm{p}}=4701 /(\mathrm{mol} \cdot \mathrm{s}), \mathrm{W}=10^{-8}(1, \mathrm{~N}=3818)$, $10^{-7}(2, \mathrm{~N}=1221), 10^{-6}(3, \mathrm{~N}=424) \mathrm{mol} / 1, \mathrm{k}_{\mathrm{tc}}=8 \cdot 10^{6} 1 /(\mathrm{mol} \cdot \mathrm{s})$ and $\mathrm{k}_{\mathrm{td}}=2 \cdot 4 \cdot 10^{7} 1 /(\mathrm{mol} \cdot \mathrm{s})$. For dashed curves $\mathrm{k}_{\mathrm{tc}}=0$ and $\mathrm{k}_{\mathrm{td}}=3.2 \cdot 10^{7} 1 /(\mathrm{mol} \cdot \mathrm{s})$.

concentration increases whereas the changes in distributions are insignificant at the region of long chains. High-molecular peaks (i $>250$ ) in the distribution calculated at $\left[\mathrm{Z}_{0}\right]=0$ (curve 1) are retained under initial inhibitor concentration in the beginning of pulse-periodic irradiation (curves 2, 3 and 4). The values i corresponding to extrema and inflection points in $B_{i}$ distributions $(\mathrm{Tab} .1, \mathrm{c}$ ) remain parctically intact with increasing of inhibitor initial concentration. Thus the existence of some initial concentration of inhibitor burned during the polymerization process has a slight influence on accuracy by polymerization rate constant determination.

\section{CONCLUSIONS}

In this paper expressions for calculation of polymer molecular distributions under polymerization initiation by arbitrary sequence of $\delta$-shape pulses and with inhibitor within the polymerizing medium are presented. The expressions obtained at $[\mathrm{Z}]=$ const can be 
used for analysis of MWDs in case of linear termination of growing chain caused detachment of hydrogen atom, cyclization of growing polymer chains, for example, and other mechanisms of linear termination not resulting in the occurrence of polymerization active centers.

Such linear termination as the chain transfer to monomer results in appearance of polymerization active centers. Qualitatively this process can be modeled by increasing of initiating pulses. It has shown in paper $^{11}$ that with increasing of rectangular pulse duration the intensity of MWDs low-molecular region increases simultaneously with intensity decreasing in the high-molecular region. Thus at the same pulses repetition rate the increase in their duration causes decreasing of high-molecular peaks intensity in the MWDs spectra.

Under polymerization with high concentration of retarder the PLP method confidently can be used at $\tau>\mathrm{T}$ and it is likely that no separate peak due to pulse-periodic irradiation will be observed in the MWDs spectra at $\tau<\mathrm{T} / 3$ because of too low concentration of radicals with chain length $\mathrm{i}>\mathrm{UT} / 3$. If the polymerization takes place after inhibitor burning as a rule (i.e. at the presence of induction period) then the PLP method can be applied with confidence because the MWDs spectrum changes insignificantly as against to that without inhibitor in the system (apart from the region of low i).

The fact that in some experiments on determination of $k_{p}$ value only one peak or its absence is found in the MWDs spectrum can be associated either with chains linear termination in the polymerizing medium (for example, due to retardation) or with non-optimal parameters of pulse-periodic irradiation (repetition rate and energy density of initiating pulses). Figure 5 shows distributions $B_{i}$ and $i B_{i}$ calculated for methylmethacrylate polymerization by pulses sequence with different energy density. The estimations have shown that both the spectra contrast and the peak number increase with decreasing of initiating radical concentration.

\section{References}

1. O. F. Olaj, I. Bitai and F. Hinkelmann, Macrom. Chem. 188, 1689 (1987).

2. T. P. Davis, J. Photocem. Photobio. A: Chem. 77, 1 (1994).

3. R. A. Hutchinson, J. R. Richards and M. T. Aronson, Macromolecules 27, 4530 (1994).

4. T. P. Davis, K. F. O'Driscoll, M. C. Piton and M. A. Winnik, Macromolecules 22, 2785 (1989).

5. T. P. Davis, K. F. O'Driscoll, M. C. Piton and M. A. Winnik, J. Polym. Sci., Polym. Lett. Ed. 27, 181 (1989).

6. T. P. Davis, K. F. O'Driscoll, M. C. Piton and M. A. Winnik, Macromolecules 23, 2113 (1990).

7. T. P. Davis, K. F. O'Driscoll, M. C. Piton and M. A. Winnik, Polym. Int. 24, 65 (1991).

8. C. E. Hoyle, M. A. Trapp and C. H. Chang, J. Polym. Sci. Polym. Chem. Ed. 27, 1609 (1989).

9. C. E. Hoyle, M. A. Trapp and C. H. Chang, ACS. Polym. Mater. Sci. Eng. 57, 570 (1987).

10. P. O. Danis, D. E. Karr, D. G. Westmoreland, M. C. Piton, D. I. Chrisite, P. A. Clay, S. H. Kable and R. G. Gilbert, Macromolecules 26, 6684 (1994).

11. A. V. Evseev and A. N. Nikitin, Laser Chem., in press.

A. V. Evseev and A. N. Nikitin, Abstracts, 35th IUPAC Intern. Symp. on Macromolecules, University of Akron, Akron, Ohio, USA, 1994.

12. S. I. Kuchanov, Methods of kinetic calculations in chemistry of polymers (in Russian), M., Khimia, (1978).

13. R. A. Hutchinson, M. T. Aronson and R. Richards, Macromolecules 26, 6410 (1993). 